\title{
On the number of representations of integers as the sum of $k$ terms
}

\author{
by
}

SÁNDOR Z. KISS (Budapest)

1. Introduction. Let $\mathbb{N}$ denote the set of positive integers. Let $k>2$ be a fixed integer and let $\mathcal{A}=\left\{a_{1}, a_{2}, \ldots\right\}\left(a_{1}<a_{2}<\cdots\right)$ be an infinite sequence of positive integers. For $n=1,2, \ldots$ let $R_{k}(n)$ denote the number of solutions of $a_{i_{1}}+\cdots+a_{i_{k}}=n, a_{i_{1}} \in \mathcal{A}, \ldots, a_{i_{k}} \in \mathcal{A}$. For $k=2$, P. Erdôs and A. Sárközy studied how regular the behaviour of the function $R_{2}(n)$ can be. In [2] they proved the following theorem:

THEOREM 1. If $F(n)$ is an arithmetic function such that

$$
\begin{gathered}
F(n) \rightarrow \infty, \\
F(n+1) \geq F(n) \quad \text { for } n \geq n_{0}, \\
F(n)=o\left(\frac{n}{(\log n)^{2}}\right),
\end{gathered}
$$

and we write

$$
\Delta(N)=\sum_{n=1}^{N}\left(R_{2}(n)-F(n)\right)^{2},
$$

then

$$
\Delta(N)=o(N F(N))
$$

cannot hold.

In [3] they showed that the above result is nearly best possible:

THEOREM 2. If $F(n)$ is an arithmetic function satisfying

$$
F(n)>36 \log n \quad \text { for } n>n_{0},
$$

and there exists a real function $g(x)$, defined for $0<x<\infty$, and real numbers $x_{0}, n_{1}$ such that

2010 Mathematics Subject Classification: Primary 11B34.

Key words and phrases: additive number theory, general sequences, additive representation function. 
(i) $g^{\prime}(x)$ exists and it is continuous for $0<x<\infty$,

(ii) $g^{\prime}(x) \leq 0$ for $x \geq x_{0}$,

(iii) $0<g(x)<1$ for $x \geq x_{0}$,

(iv) $\left|F(n)-2 \int_{0}^{n / 2} g(x) g(n-x) d x\right|<(F(n) \log n)^{1 / 2}$ for $n>n_{1}$,

then there exists a sequence $\mathcal{A}$ such that

$$
\left|R_{2}(n)-F(n)\right|<8(F(n) \log n)^{1 / 2} \quad \text { for } n>n_{2} .
$$

In [6] G. Horváth extended Theorem 1 to any $k>2$ :

THEOREM 3. If $F(n)$ is an arithmetic function such that

$$
\begin{gathered}
F(n) \rightarrow \infty, \\
F(n+1) \geq F(n) \quad \text { for } n \geq n_{0}, \\
F(n)=o\left(\frac{n}{(\log n)^{2}}\right),
\end{gathered}
$$

and we write

$$
\Delta(N)=\sum_{n=1}^{N}\left(R_{k}(n)-F(n)\right)^{2}
$$

then

$$
\Delta(N)=o(N F(N))
$$

cannot hold.

A. Sárközy proposed to prove an analogue of Theorem 2 for $k>2[8$, Problem 3]. In this paper my goal is to extend Theorem 2 to any $k>2$, i.e., to show that Theorem 3 is nearly best possible. In fact, I will prove the following theorem:

THEOREM 4. If $k>2$ is a positive integer, $c_{8}$ is a constant large enough in terms of $k, F(n)$ is an arithmetic function satisfying

$$
F(n)>c_{8} \log n \quad \text { for } n>n_{0},
$$

and there exists a real function $g(x)$, defined for $0<x<\infty$, and real numbers $x_{0}, n_{1}$ and constants $c_{7}, c_{9}$ such that

$$
\begin{aligned}
& \text { (i) } 0<g(x) \leq \frac{(\log x)^{1 / k}}{x^{1-(k+1) / k^{2}}}<1 \text { for } x \geq x_{0}, \\
& \text { (ii) }\left|F(n)-k ! \sum_{\substack{x_{1}+\cdots+x_{k}=n \\
1 \leq x_{1}<\cdots<x_{k}<n}} g\left(x_{1}\right) \ldots g\left(x_{k}\right)\right|<c_{7}(F(n) \log n)^{1 / 2} \text { for } n>n_{1},
\end{aligned}
$$

then there exists a sequence $\mathcal{A}$ such that

$$
\left|R_{k}(n)-F(n)\right|<c_{9}(F(n) \log n)^{1 / 2} \quad \text { for } n>n_{2} .
$$


It is easy to see that the following functions satisfy the conditions of Theorem 4: $g(x)=c_{10}\left((\log x)^{\beta} / x^{\alpha}\right)$, where $c_{10}$ is a positive constant, $\alpha>$ $1-(k+1) / k^{2}$, or $\alpha=1-(k+1) / k^{2}$ and $\beta \leq 1 / k$. It follows that for $F(n)=n^{\delta}(\log n)^{\gamma}$ with $0<\delta \leq 1 / k$, or $0 \leq \gamma<1$ there is a sequence $\mathcal{A}$ for which $R_{k}(n)$ satisfies the conclusion of the theorem. For $k=2$ in [3] $\mathrm{P}$. Erdôs and A. Sárközy used the probabilistic method to construct $\mathcal{A}$. In the case $k=2$, certain events in their paper were mutually independent. For $k>2$ the independence fails, thus to prove Theorem 4 we need deeper probabilistic tools.

2. Probabilistic tools. The proof of Theorem 4 is based on the probabilistic method due to Erdôs and Rényi. There is an excellent summary of this method in Halberstam and Roth's book [5]. We use the notation and terminology of that book. First we give a survey of the probabilistic tools and notation we use in the proof of Theorem 4. Let $\Omega$ denote the set of strictly increasing sequences of positive integers. In this paper we denote the probability of an event $E$ by $P(E)$.

Lemma 1. Let

$$
\alpha_{1}, \alpha_{2}, \ldots
$$

be real numbers satisfying

$$
0 \leq \alpha_{n} \leq 1 \quad(n=1,2, \ldots) .
$$

Then there exists a probability space $(\Omega, S, P)$ with the following two properties:

(i) For every natural number $n$, the event $E^{(n)}=\{\mathcal{A} \in \Omega: n \in \mathcal{A}\}$ is measurable, and $P\left(E^{(n)}\right)=\alpha_{n}$.

(ii) The events $E^{(1)}, E^{(2)}, \ldots$ are independent.

See $[5$, Theorem 13, p. 142]. We denote the characteristic function of the event $E^{(n)}$ by $\varrho(\mathcal{A}, n)$ :

$$
\varrho(\mathcal{A}, n)= \begin{cases}1 & \text { if } n \in \mathcal{A}, \\ 0 & \text { if } n \notin \mathcal{A} .\end{cases}
$$

Furthermore, we denote the number of solutions of $a_{i_{1}}+\cdots+a_{i_{k}}=n$ by $r_{k}(n)$, where $a_{i_{1}} \in \mathcal{A}, \ldots, a_{i_{k}} \in \mathcal{A}, 1 \leq a_{i_{1}}<\cdots<a_{i_{k}}<n$. Thus

$$
r_{k}(n)=\sum_{\substack{\left(a_{1}, \ldots, a_{k}\right) \in \mathbb{N}^{k} \\ 1 \leq a_{1}<\cdots<a_{k}<n \\ a_{1}+\cdots+a_{k}=n}} \varrho\left(\mathcal{A}, a_{1}\right) \ldots \varrho\left(\mathcal{A}, a_{k}\right) .
$$

Let $r_{k}^{*}(n)$ denote the number of those representations $n=a_{i_{1}}+\cdots+a_{i_{k}}$ in which there are at least two equal terms. Thus

$$
R_{k}(n)=k ! r_{k}(n)+r_{k}^{*}(n) .
$$


It is easy to see from (3) that $r_{k}(n)$ is a sum of random variables. However, for $k>2$ these variables are not independent because the same $\varrho\left(\mathcal{A}, a_{i}\right)$ may appear in many terms; therefore we need deeper probabilistic tools.

Our proof is based on a method of J. H. Kim and V. H. Vu. In the next section we give a short survey of their method. The interested reader can find more details in [7], [9], [10]. Assume that $t_{1}, \ldots, t_{n}$ are independent binary (i.e., $\{0,1\}$-valued) random variables. Consider a polynomial $Y$ in $t_{1}, \ldots, t_{n}$ of degree $k$. We say $Y$ is positive if it can be written in the form $Y=\sum_{i} e_{i} \Gamma_{i}$, where the $e_{i}$ 's are positive and each $\Gamma_{i}$ is a product of some $t_{j}$ 's. Given a (multi-) set $A, \partial_{A}(Y)$ denotes the partial derivative of $Y$ with respect to the variables with indices in $A$. For instance, if $Y=t_{1} t_{2}^{2}$ and $A_{1}=\{1,2\}$ and $A_{2}=\{2,2\}$ then $\partial_{A_{1}}(Y)=2 t_{2}$ and $\partial_{A} Y=2 t_{1}$. If $A$ is empty then $\partial_{A}(Y)=Y$. Let $E_{A}(Y)$ denote the expectation of $\partial_{A}(Y)$. Furthermore, set

$$
E_{j}(Y)=\max _{|A| \geq j} E_{A}(Y) \quad \text { for } j=0,1, \ldots, k,
$$

so $E_{0}(Y)=E(Y)$.

TheOREM $5(\mathrm{Kim}-\mathrm{Vu})$. For every positive integer $k$ there are positive constants $d_{k}$ and $b_{k}$ depending only on $k$ such that for any positive polynomial $Y=Y\left(t_{1}, \ldots, t_{n}\right)$ of degree $k$, where the $t_{i}$ 's are independent binary random variables,

$$
P\left(|Y-E(Y)| \geq d_{k} \lambda^{k} \sqrt{E_{0}(Y) E_{1}(Y)}\right) \leq b_{k} e^{-\lambda / 4+(k-1) \log n} .
$$

See [7] for the proof. Finally, we need the Borel-Cantelli lemma (see [5]):

Lemma 2. Let $\left\{B_{i}\right\}$ be a sequence of events in a probability space. If

$$
\sum_{j=1}^{\infty} P\left(B_{j}\right)<\infty,
$$

then with probability 1 , at most a finite number of the events $B_{j}$ can occur.

3. Proof of Theorem 4. Fix a number $n$ and write

$$
S_{n}=\left\{\left(a_{1}, \ldots, a_{k}\right) \in \mathbb{N}^{k}: 0<a_{1}<\cdots<a_{k}<n, a_{1}+\cdots+a_{k}=n\right\} .
$$

Define a sequence (1) of real numbers by

$$
\alpha_{n}= \begin{cases}g(n) & \text { if } n \geq x_{0}, \\ 0 & \text { otherwise }\end{cases}
$$

and let $(\Omega, S, P)$ be the probability space as in Lemma 1 . Clearly the sequence $\alpha_{n}$ satisfies (2). Thus

$$
r_{k}(n)=\sum_{\left(a_{1}, \ldots, a_{k}\right) \in S_{n}} t_{a_{1}} \ldots t_{a_{k}}
$$


where

$$
t_{a_{i}}= \begin{cases}1 & \text { if } a_{i} \in \mathcal{A} \\ 0 & \text { if } a_{i} \notin \mathcal{A}\end{cases}
$$

Then we have

$$
\lambda_{n}=E\left(r_{k}(n)\right)=\sum_{\left(a_{1}, \ldots, a_{k}\right) \in S_{n}} P\left(a_{1} \in \mathcal{A}\right) \ldots P\left(a_{k} \in \mathcal{A}\right),
$$

where $E(\zeta)$ denotes the expectation of the random variable $\zeta$. To prove Theorem 4 we will give an upper estimate for $\left|R_{k}(n)-k ! \lambda_{n}\right|$. As $\mathrm{Vu}$ in [10], we split $r_{k}(n)$ into two parts, as follows. Let $a$ be a small positive constant, say $a<1 / 2(k+1)$, and let $S_{n}^{[1]}$ be the subset of all $\left(a_{1}, \ldots, a_{k}\right) \in S_{n}$ with $a_{1} \geq n^{a}$, and $S_{n}^{[2]}=S_{n} \backslash S_{n}^{[1]}$. We split $r_{k}(n)$ into the sum of two terms corresponding to $S_{n}^{[1]}$ and $S_{n}^{[2]}$, respectively:

$$
r_{k}(n)=r_{k}^{[1]}(n)+r_{k}^{[2]}(n),
$$

where

$$
r_{k}^{[j]}(n)=\sum_{\left(a_{1}, \ldots, a_{k}\right) \in S_{n}^{[j]}} t_{a_{1}} \ldots t_{a_{k}}
$$

and set

$$
\lambda_{n}^{[j]}=E\left(r_{k}^{[j]}(n)\right) .
$$

Clearly

$$
\begin{aligned}
\left|R_{k}(n)-k ! \lambda_{n}\right| & \leq\left|R_{k}(n)-k ! r_{k}(n)\right|+k !\left|r_{k}(n)-\lambda_{n}\right| \\
& =r_{k}^{*}(n)+k !\left|r_{k}^{[1]}(n)+r_{k}^{[2]}(n)-\lambda_{n}^{[1]}-\lambda_{n}^{[2]}\right| \\
& \leq r_{k}^{*}(n)+k !\left|r_{k}^{[1]}(n)-\lambda_{n}^{[1]}\right|+k !\left|r_{k}^{[2]}(n)-\lambda_{n}^{[2]}\right| \\
& =r_{k}^{*}(n)+I_{1}+I_{2} .
\end{aligned}
$$

The rest of the proof of Theorem 4 has four parts. In the first part we estimate $I_{1}$, in the second $I_{2}$, in the third $r_{k}^{*}(n)$, and in the last part we complete the proof.

Estimating $I_{1}$. We will apply Theorem 5 so we need an upper bound for $E_{1}\left(r_{k}^{[1]}(n)\right)$. To do this, it is clear from the definition of $E_{1}$ that we need the following lemma, which guarantees that every partial derivative of $r_{k}^{[1]}(n)$ has small expectation.

Lemma 3. For all non-empty multi-sets $A$ of size at most $k-1$,

$$
E\left(\partial_{A}\left(r_{k}^{[1]}(n)\right)\right)=O\left(n^{-a / 2 k^{2}}\right) .
$$

Proof. This can be proved similarly to Lemma 5.3 in [10]. For completeness I will present the proof. Consider a multi-set $A$ of $k-l$ elements and 
$\sum_{x \in A} x=n-m$. There exists a constant $c(k)$ such that

$$
\partial_{A}\left(r_{k}^{[1]}(n)\right) \leq c(k) \sum_{\substack{n^{a}<a_{1}<\cdots<a_{l} \\ a_{1}+\cdots+a_{l}=m}} t_{a_{1}} \ldots t_{a_{l}} .
$$

As $a_{l} \geq m / l$ and $\sum_{x=1}^{m} x^{1 / k-1} \approx \int_{1}^{m} z^{1 / k-1} d z \approx m^{1 / k}$, and using assumption (i) of Theorem 4, we have

$$
\begin{aligned}
& E\left(\partial_{A}\left(r_{k}^{[1]}(n)\right)\right) \\
& =O\left(\sum_{\substack{n^{a}<a_{1}<\ldots<a_{l} \\
a_{1}+\cdots+a_{l}=m}} P\left(a_{1} \in \mathcal{A}\right) \ldots P\left(a_{l} \in \mathcal{A}\right)\right)=O\left(\sum_{\substack{n^{a}<a_{1}<\ldots<a_{l} \\
a_{1}+\cdots+a_{l}=m}} g\left(a_{1}\right) \ldots g\left(a_{l}\right)\right) \\
& =O(\log n) \sum_{\substack{n^{a}<a_{1}<\cdots<a_{l} \\
a_{1}+\cdots+a_{l}=m}} a_{1}^{(k+1) / k^{2}-1} \ldots a_{l}^{(k+1) / k^{2}-1} \\
& =O(\log n) O\left(\left(\sum_{x=1}^{m} x^{(k+1) / k^{2}-1}\right)^{l-1}(m / l)^{(k+1) / k^{2}-1}\right) \\
& =O(\log n) O\left(m^{(l-1)(k+1) / k^{2}}(m / l)^{(k+1) / k^{2}-1}\right) \\
& =O(\log n) O\left(m^{\left(l(k+1)-k^{2}\right) / k^{2}}\right)=O\left(n^{-a / 2 k^{2}}\right) \text {, }
\end{aligned}
$$

since $k-1 \geq l$ and $m \geq n^{a}$. The proof of Lemma 3 is complete.

By the definition of $E_{1}\left(r_{k}^{[1]}(n)\right)$, and from Lemma 3, it is clear that $E_{1}\left(r_{k}^{[1]}(n)\right)=\max _{|A| \geq 1} E_{A}\left(r_{k}^{[1]}(n)\right) \leq c n^{-a / 2 k^{2}}$, where $c$ is a constant. It is clear from (5) that $r_{k}^{[1]}(n)$ is a positive polynomial of degree $k$. Now we apply Theorem 5 with $\lambda=\left(\log n / E_{1}\left(r_{k}^{[1]}(n)\right)\right)^{1 / 2 k}$. If $n$ is large enough we have

$$
\begin{aligned}
& P\left(\left|r_{k}^{[1]}(n)-\lambda_{n}^{[1]}\right| \geq d\right.\left.\sqrt{\frac{\log n}{E_{1}\left(r_{k}^{[1]}(n)\right)}} \sqrt{\lambda_{n}^{[1]} E_{1}\left(r_{k}^{[1]}(n)\right)}\right) \\
& \leq b_{k} \exp \left(-\frac{1}{4} \sqrt[2 k]{\frac{\log n}{E_{1}\left(r_{k}^{[1]}(n)\right)}}+(k-1) \log n\right) \\
& \leq b_{k} \exp \left(-\frac{1}{4} \sqrt[2 k]{\frac{\log n}{n^{-a / 2 k^{2}}}}+(k-1) \log n\right) \\
&<\exp (-2 \log n)=\frac{1}{n^{2}} .
\end{aligned}
$$

Applying the above result we obtain

$$
\sum_{n=1}^{\infty} P\left(\left|r_{k}^{[1]}(n)-\lambda_{n}^{[1]}\right| \geq d_{k} \sqrt{\lambda_{n}^{[1]} \log n}\right)<\sum_{n=1}^{\infty} \frac{1}{n^{2}}<\infty .
$$


By the Borel-Cantelli lemma, with probability 1 , there exists $n_{0}$ such that

$$
\left|r_{k}^{[1]}(n)-\lambda_{n}^{[1]}\right|<d_{k} \sqrt{\lambda_{n}^{[1]} \log n} \text { for } n>n_{0} .
$$

Estimating $I_{2}$. We will prove similarly to the proof in [10] that for almost every sequence $\mathcal{A}$, there is a finite number $c_{11}(\mathcal{A})$ such that $r_{k}^{[2]}(n) \leq c_{11}(\mathcal{A})$ for all sufficiently large $n$. Let $r_{l}(n)$ denote the number of representations of $n$ as the sum of $l$ distinct numbers from $\mathcal{A}$. First we estimate $E\left(r_{l}(n)\right)$ similarly to [4]. Fix $2 \leq l \leq k-1$. As $n / l<a_{l}$, by assumption (i) of Theorem 4 , we have

$$
\begin{aligned}
& E\left(r_{l}(n)\right) \leq \sum_{\substack{a_{1}+\cdots+a_{l}=n \\
1 \leq a_{1}<\cdots<a_{l}<n}} P\left(a_{1} \in \mathcal{A}\right) \ldots P\left(a_{l} \in \mathcal{A}\right) \\
& <\sum_{\substack{a_{1}+\cdots+a_{l}=n \\
1 \leq a_{1}<\cdots<a_{l}<n}} g\left(a_{1}\right) \ldots g\left(a_{l}\right) \\
& \leq \sum_{\substack{a_{1}+\cdots+a_{l}=n \\
1 \leq a_{1}<\cdots<a_{l}<n}} \frac{\left(\log a_{1}\right)^{1 / k}}{a_{1}^{1-(k+1) / k^{2}}} \cdots \frac{\left(\log a_{l}\right)^{1 / k}}{a_{l}^{1-(k+1) / k^{2}}} \\
& =n^{o(1)} \sum_{\substack{a_{1}+\cdots+a_{l}=n \\
1 \leq a_{1}<\cdots<a_{l}<n}} \frac{1}{\left(a_{1} \ldots a_{l}\right)^{1-(k+1) / k^{2}}} \\
& \leq n^{o(1)}\left(n^{(k+1) / k^{2}-1+o(1)} \sum_{\substack{a_{1}+\cdots+a_{l}=n \\
1 \leq a_{1}<\cdots<a_{l}<n}} \frac{1}{\left(a_{1} \ldots a_{l-1}\right)^{1-(k+1) / k^{2}}}\right) \\
& \leq n^{(k+1) / k^{2}-1+o(1)} \sum_{\substack{1 \leq a_{i} \leq n \\
i=1, \ldots, l-1}} \frac{1}{\left(a_{1} \ldots a_{l-1}\right)^{1-(k+1) / k^{2}}} \\
& =n^{(k+1) / k^{2}-1+o(1)}\left(\sum_{1 \leq a_{1} \leq n} \frac{1}{a_{1}^{1-(k+1) / k^{2}}}\right)^{l-1} \\
& =n^{(k+1) / k^{2}-1+o(1)}\left(n^{(k+1) / k^{2}+o(1)}\right)^{l-1}=n^{-1+l(k+1) / k^{2}+o(1)} \text {. }
\end{aligned}
$$

Let $T_{1}=\left\{a_{1}, \ldots, a_{k}\right\}, T_{2}=\left\{b_{1}, \ldots, b_{k}\right\}, T_{1} \neq T_{2}, T_{1}, T_{2} \subset \mathcal{A}$ and

$$
a_{1}+\cdots+a_{k}=b_{1}+\cdots+b_{k}=n .
$$

We say these representations are disjoint if they share no element in common. Let $f_{l}(n)$ denote the maximum number of pairwise disjoint representations of $n$ as the sum of $l$ distinct numbers from $\mathcal{A}$. We show that with probability $1, f_{l}(n)$ is bounded. We will apply the following result due to Erdôs and Tetali which is called the disjointness lemma. We say events $G_{1}, \ldots, G_{n}$ are independent if for all subsets $I \subseteq\{1, \ldots, n\}, P\left(\bigcap_{i \in I} G_{i}\right)=\prod_{i \in I} P\left(G_{i}\right)$. 
Lemma 4. If $\sum_{i} P\left(B_{i}\right) \leq \mu$, then

$$
\sum_{\substack{\left(B_{1}, \ldots, B_{l}\right) \\ \text { independent }}} P\left(B_{1} \cap \cdots \cap B_{l}\right) \leq \mu^{l} / l ! .
$$

Proof. This is Lemma 1 in [4]. Let

$$
\mathcal{B}=\left\{\left(a_{1}, \ldots, a_{l}\right) \in \mathcal{A}^{n}: a_{1}+\cdots+a_{l}=n, 1 \leq a_{1}<\cdots<a_{l}<n\right\} .
$$

Let $H(\mathcal{B})=\{\mathcal{T} \subset \mathcal{B}$ : all the $K \in \mathcal{T}$ are pairwise disjoint $\}$ and $c_{1}$ be a constant. It is clear that the pairwise disjointness of the sets implies the independence of the associated events, i.e., if $K_{1}$ and $K_{2}$ are pairwise disjoint representations, then the events $K_{1} \subset \mathcal{A}, K_{2} \subset \mathcal{A}$ are independent. Thus by (8) and Lemma 4 we have

$$
\begin{aligned}
P\left(f_{l}(n)>c_{1}\right) \leq P\left(\bigcup_{\substack{\mathcal{T} \subset H(\mathcal{B}) \\
|\mathcal{T}|=c_{1}+1}} \bigcap_{K \in \mathcal{T}} K\right) \leq \sum_{\substack{\mathcal{T} \subset H(\mathcal{B}) \\
|\mathcal{T}|=c_{1}+1}} P\left(\bigcap_{K \in \mathcal{T}} K\right) \\
=\sum_{\substack{\left(K_{1}, \ldots, K_{c_{1}+1}\right) \\
\text { pairwise } \\
\text { disjoint }}} P\left(K_{1} \cap \cdots \cap K_{c_{1}+1}\right) \leq \frac{1}{\left(c_{1}+1\right) !}\left(E\left(f_{l}(n)\right)\right)^{c_{1}+1} \\
\leq \frac{1}{\left(c_{1}+1\right) !}\left(E\left(r_{l}(n)\right)\right)^{c_{1}+1} \leq \frac{1}{\left(c_{1}+1\right) !} n^{-2+o(1)}
\end{aligned}
$$

if $c_{1}$ large enough. By the Borel-Cantelli lemma, with probability 1 for almost every random sequence $\mathcal{A}$ there is a finite number $c_{1}(\mathcal{A})$ such that for any $l<k$ and all $n$, the maximal number of disjoint $l$-representations of $n$ from $\mathcal{A}$ is at most $c_{1}(\mathcal{A})$.

In the next step we estimate $E\left(r_{k}^{[2]}(n)\right)$ as in Lemma 3. Using also the fact that $\sum_{x=1}^{m} x^{1 / k-1} \approx \int_{1}^{m} z^{1 / k-1} d z \approx m^{1 / k}$, and $a_{k} \geq n / k, a<1 /(2(k+1))$, and (i) of Theorem 4, we have

$$
\begin{aligned}
E\left(r_{k}^{[2]}(n)\right) & =E\left(\sum_{\left(a_{1}, \ldots, a_{k}\right) \in S_{n}^{[2]}} t_{a_{1}} \ldots t_{a_{k}}\right) \\
& =O\left(\sum_{\left(a_{1}, \ldots, a_{k}\right) \in S_{n}^{[2]}} P\left(a_{1} \in \mathcal{A}\right) \ldots P\left(a_{k} \in \mathcal{A}\right)\right) \\
& =O(\log n) \sum_{\substack{a_{1}+\ldots+a_{k}=n \\
a_{1} \leq n^{k}}} a_{1}^{(k+1) / k^{2}-1} \ldots a_{k}^{(k+1) / k^{2}-1}
\end{aligned}
$$




$$
\begin{aligned}
& =O(\log n) O\left(\sum_{x=1}^{n^{a}} x^{(k+1) / k^{2}-1}\left(\sum_{x=1}^{n} x^{(k+1) / k^{2}-1}\right)^{k-2}(n / k)^{(k+1) / k^{2}-1}\right) \\
& =O\left(n^{(a(k+1)-1) / k^{2}} \log n\right)=O\left(n^{-1 / 2 k^{2}}\right) .
\end{aligned}
$$

Thus by Lemma 4 and the Borel-Cantelli lemma, with probability 1, there is a constant $c_{2}$ such that almost surely the maximum number of disjoint representations of $n$ in $r_{k}^{[2]}(n)$ is at most $c_{2}$ for all large $n$.

To finish the proof it suffices to show that $r_{k}^{[2]}(n)$ is bounded by a constant. The proof is purely combinatorial. We need the following well-known result due to Erdős and Rado [1]. Let $r$ be a positive integer, $r \geq 3$. A collection of sets $D_{1}, \ldots, D_{r}$ forms a $\Delta$-system if the sets have pairwise the same intersection.

LEMMA 5. If $H$ is a collection of sets of size at most $k$ and $|H|>$ $(r-1)^{k} k$ ! then $H$ contains $r$ sets forming a $\Delta$-system.

Set $C(\mathcal{A})=\left(\max \left(c_{1}(\mathcal{A}), c_{2}\right)\right)^{k} k$ ! and assume that $n$ is sufficiently large. To each representation of $n$ counted in $r_{k}^{[2]}(n)$ we assign the set formed by the $k$ terms occurring in this representation. We will apply Lemma 5 with $H$ being the collection of these sets. It is clear that if $r_{k}^{[2]}(n)>C(\mathcal{A})$, then by Lemma $5, r_{k}^{[2]}(n)$ contains a $\Delta$-system with $c_{3}=\max \left(c_{1}(\mathcal{A}), c_{2}\right)+1$ sets. If the intersection of these sets is empty, then they form a family of $c_{3}$ disjoint $k$-representations of $n$, which contradicts the definition of $c_{3}$. Otherwise, assume that the intersection of these sets is $\left\{y_{1}, \ldots, y_{j}\right\}$, where $1 \leq j \leq k-1$ and $\sum_{i=1}^{j} y_{i}=m$. Removing the common intersection of these sets we can find $c_{1}(\mathcal{A})+1(k-j)$-representations of $n-m=n-\sum_{i=1}^{j} y_{i}$. These $c_{1}(\mathcal{A})+1$ sets are disjoint due to the definition of the $\Delta$-system. Therefore in both cases we obtain a contradiction.

Estimating $r_{k}^{*}(n)$. If we collect the equal terms, we have

$$
u_{1} a_{1}+u_{2} a_{2}+\cdots+u_{h} a_{h}=n,
$$

where the $u_{i}$ 's are positive integers, and

$$
u_{1}+u_{2}+\cdots+u_{h}=k .
$$

Thus $r_{k}^{*}(n)$ denotes the number of representations (10) of $n$, where the $a_{i}$ 's are different. It can be proved similarly to the estimate of $r_{k}^{[2]}(n)$ that $r_{k}^{*}(n)$ is also bounded by a constant. For completeness we sketch the proof leaving the details to the reader. Fix $2 \leq h \leq k-1$. For fixed $u_{1}, \ldots, u_{h}$ let $s_{h}(n)$ denote the number of representations (10) of $n$. We show that $s_{h}(n)$ is bounded by a constant. (Note that we have already proved this when all $u_{i}$ 's are equal to one, and $h=k$.) First we estimate $E\left(s_{h}(n)\right)$, with a calculation similar 
to (8). Using the definition of $s_{h}(n)$, and $n / h<a_{h}$, we have

$$
\begin{aligned}
E\left(s_{h}(n)\right) & \leq \sum_{\substack{u_{1} a_{1}+\cdots+u_{h} a_{h}=n \\
1 \leq a_{1}<\cdots<a_{h}<n}} P\left(a_{1} \in \mathcal{A}\right) \ldots P\left(a_{h} \in \mathcal{A}\right) \\
& =\sum_{\substack{u_{1} a_{1}+\cdots+u_{h} a_{h}=n \\
1 \leq a_{1}<\cdots<a_{h}<n}} g\left(a_{1}\right) \ldots g\left(a_{h}\right) \\
& \leq \sum_{\substack{u_{1} a_{1}+\cdots+u_{h} a_{h}=n \\
1 \leq a_{1}<\cdots<a_{h}<n}} \frac{\left(\log a_{1}\right)^{1 / k}}{1-(k+1) / k^{2}} \cdots \frac{\left(\log a_{h}\right)^{1 / k}}{a_{h}^{1-(k+1) / k^{2}}} \\
& =n^{-1+h(k+1) / k^{2}+o(1)} .
\end{aligned}
$$

Let $s_{h}^{*}(n)$ denote the size of a maximal collection of pairwise disjoint representations (10). The same argument as in (9) shows that almost always there exists a constant $v_{h}$ such that $s_{h}^{*}(n)<v_{h}$ for $n$ large enough. In view of (12), and applying Lemma 4, we have

$$
P\left(s_{h}^{*}(n)>v_{h}\right)<n^{-2+o(1)}
$$

if $v_{h}$ is large enough. Thus by the Borel-Cantelli lemma, with probability 1 , $s_{h}^{*}(n)<v_{h}$ for every large enough $n$. We say that an $m$-tuple $\left(a_{1}, \ldots, a_{m}\right)$ $(m \leq h)$ is an $m$-representation of $n$ in the form (10) if there is a permutation $\pi$ of $\{1, \ldots, h\}$ such that $\sum_{i=1}^{m} u_{\pi(i)} a_{i}=n$. For all $m<h$, let $s_{m}^{*}(n)$ denote the size of a maximal collection of pairwise disjoint such representations of $n$. The same argument as above shows that almost always there exists a constant $p_{m}$ such that $s_{m}^{*}(n)<p_{m}$ for every large enough $n$.

In the last step we apply Lemma 5 to prove that $s_{h}(n)$ is bounded by a constant. Let $C=\left(\max \left(p_{m} h !, v_{h}\right)\right)^{h} h$ !. Let $H$ in Lemma 5 be the collection of representations (10) of $n$. Clearly $|H|=s_{h}(n)$. If $s_{h}(n)>C$, and $n$ is sufficiently large then by Lemma $5, H$ contains a $\Delta$-system with $C+1$ sets. If the intersection of these sets is empty, then they form a family of disjoint $h$-representations (10). Otherwise, let the common intersection of the sets be $\left\{y_{1}, \ldots, y_{s}\right\}$, where $1 \leq s \leq h-1$. By the pigeon-hole principle there exists a permutation $\pi$ of $\{1, \ldots, h\}$ such that we can find $p_{m}+1(k-s)$ representations of $n^{\prime \prime}=n-\sum_{i=1}^{s} u_{\pi(i)} y_{s}$. These $p_{m}+1$ sets are disjoint, thus in both cases we obtain a contradiction. Since there are only a finite number of partitions of $k$ in the form (11), we conclude that $r_{k}^{*}(n)$ is bounded by a constant, i.e., there exists a constant $C_{3}$ such that $r_{k}^{*}(n)<C_{3}$. Let $c_{4}, c_{5}, c_{6}$ be constants. Thus by (6) and (7) we have

$$
\begin{aligned}
\left|R_{k}(n)-k ! \lambda_{n}\right| & \leq\left|R_{k}(n)-k ! r_{k}(n)\right|+k !\left|r_{k}(n)-\lambda_{n}\right| \\
& <C_{3}+k !\left|r_{n}^{[1]}+r_{n}^{[2]}-\lambda_{n}^{[1]}-\lambda_{n}^{[2]}\right|
\end{aligned}
$$




$$
\begin{aligned}
& \leq C_{3}+k !\left|r_{n}^{[1]}-\lambda_{n}^{[1]}\right|+k !\left|r_{n}^{[2]}-\lambda_{n}^{[2]}\right| \\
& \leq C_{3}+d_{k} k ! \sqrt{\lambda_{n}^{[1]} \log n}+2 k ! c_{4} \leq c_{5}+d_{k} k ! \sqrt{\lambda_{n} \log n} .
\end{aligned}
$$

End of proof. We argue as in [3]. In view of the estimate above and assumption (ii), for large $n$ we have

$$
\begin{aligned}
& \left|R_{k}(n)-F(n)\right| \leq\left|R_{k}(n)-k ! \lambda_{n}\right|+\left|k ! \lambda_{n}-F(n)\right| \\
& \quad<c_{5}+d_{k} k !\left(\lambda_{n} \log n\right)^{1 / 2}+\left|k ! \lambda_{n}-F(n)\right| \\
& \leq c_{5}+c_{6}\left(\left(\frac{1}{k !} F(n)+\frac{1}{k !}\left|k ! \lambda_{n}-F(n)\right|\right) \log n\right)^{1 / 2}+\left|k ! \lambda_{n}-F(n)\right| \\
& \quad<c_{5}+c_{6}\left(\left(\frac{1}{k !} F(n)+\frac{c_{7}}{k !}(F(n) \log n)^{1 / 2}\right) \log n\right)^{1 / 2}+c_{7}(F(n) \log n)^{1 / 2} \\
& <c_{5}+c_{6}\left(\left(\frac{1}{k !} F(n)+\frac{c_{7}}{k !}\left(F(n) \frac{F(n)}{c_{8}}\right)^{1 / 2}\right) \log n\right)^{1 / 2}+c_{7}(F(n) \log n)^{1 / 2} \\
& =c_{5}+c_{6}\left(\left(\frac{1}{k !}+\frac{c_{7}}{\sqrt{c_{8} k !}}\right) F(n) \log n\right)^{1 / 2}+c_{7}(F(n) \log n)^{1 / 2} \\
& <c_{9}(F(n) \log n)^{1 / 2} .
\end{aligned}
$$

The proof of Theorem 4 is complete.

Acknowledgements. The author would like to thank Professor András Sárközy for valuable discussions.

\section{References}

[1] P. Erdôs and R. Rado, Intersection theorems for system of sets, J. London Math. Soc. 35 (1960), 85-90.

[2] P. Erdős and A. Sárközy, Problems and results on additive properties of general sequences I, Pacific J. Math. 118 (1985), 347-357.

[3] - - - Problems and results on additive properties of general sequences II, Acta Math. Hungar. 48 (1986), 201-211.

[4] P. Erdôs and P. Tetali, Representations of integers as the sum of $k$ terms, Random Structures Algorithms 1 (1990), 245-261.

[5] H. Halberstam and K. F. Roth, Sequences, Springer, New York, 1983.

[6] G. Horváth, On an additive property of sequences of nonnegative integers, Period. Math. Hungar. 45 (2002), 73-80.

[7] J. H. Kim and V. H. Vu, Concentration of multivariate polynomials and its applications, Combinatorica 20 (2000), 417-434.

[8] A. Sárközy, On the number of additive representations of integers, in: More Sets, Graphs and Numbers, A Salute to Vera T. Sós and András Hajnal, eds. E. Györi et al., Bolyai Soc. Math. Stud. 15, J. Bolyai Math. Soc. and Springer, 2006, 329-339. 
[9] V. H. Vu, Chernoff type bounds for sums of dependent random variables and applications in additive number theory, in: Number Theory for the Millennium, III (Urbana, IL, 2000), A K Peters, Natick, MA, 2002, 341-356.

[10] -, On the concentration of multi-variate polynomials with small expectation, Random Structures Algorithms 16 (2000), 344-363.

Department of Algebra and Number Theory

Eötvös Loránd University

Pázmány Péter Sétány 1/c

H-1117 Budapest, Hungary

E-mail: kisspest@cs.elte.hu

Received on 24.11.2008

and in revised form on 27.1.2009 